\title{
Optimization of injection molding process parameters based on GA-ELM-GA
}

\author{
Yi Mei, and Maoyuan Xue* \\ School of Mechanical Engineering, Guizhou University, Guiyang 550025, China
}

\begin{abstract}
The most common optimization method for the optimization of injection mold process parameters is range analysis, but there is often a nonlinear coupling relationship between injection molding process parameters and quality indicators. Therefore, it is difficult to find the optimal process combination in range analysis. In this article, a genetic algorithm optimized extreme learning machine network model (GA-ELM) combined with genetic algorithm (GA) was proposed to optimize the process parameters of the injection mold. Take the injection molding process parameter optimization of an electrical appliance buckle cover shell as an example. In order to find the process parameters corresponding to the minimum warpage deformation, an orthogonal experiment was designed and the results of the orthogonal experiment were analyzed. Then, the corresponding optimal process combination and the degree of influence of process parameters on the warpage deformation were obtained. At the same time, the extreme learning machine network model (GA-ELM) optimized by the genetic algorithm was used to predict the warpage deformation of the plastic part. The trained GA-ELM model can map nonlinear coupling relationship between the five process parameters and the warpage deformation well. And the optimal process parameters in the trained GA-ELM network model was searched by the powerful optimization ability of genetic algorithm. Generally speaking, the warpage deformation after optimization by range analysis is reduced by $6.7 \%$ compared with the minimum warpage after optimization by orthogonal experiment. But compared to the minimum warpage deformation after orthogonal experiment optimization, the warpage deformation after GAELM-GA optimization is reduced by $22 \%$, which is better than that of the range analysis, thus verifying the feasibility and the optimization of the optimization method. This optimization method provides a certain theoretical reference and technical support for the field involving the optimization of process parameters.
\end{abstract}

Keywords: Process parameter optimization, Orthogonal test, Poor analysis, ELM, GA-ELM, GA-ELM-GA.

\footnotetext{
*Corresponding author: 1593768152@qq.com
} 


\section{Introduction}

There are many methods to optimize the process parameters of injection molded parts. Many scholars combine computer simulation technology (Moldflow) with multiple statistical experimental methods, such as orthogonal experiment, Kringing model, response surface method and Taguchi experiment, to study the internal relationship between different process parameters and the quality of injection molded parts. Therefore, these methods are used to optimize the injection molding process parameters [1-3]. However, due to the highly nonlinear mapping relationship between the injection molding quality and the injection molding process parameters, it is difficult to clearly express the coupling relationship between the injection molding process parameters and the molding quality indicators with conventional statistical experimental methods, and it also lacks the advantages of intelligence and integration, so there are certain limitations [4]. Artificial neural network has the characteristics of self-organization and self-learning, and has strong ability to fit nonlinear functions. The artificial neural network can be used to fit the injection molding process parameters and molding, and then the optimization algorithm can be used to optimize the network model, so as to get the better process parameters [5-6]. The ELM network model is widely used in many engineering fields. However, due to the random generation of ELM weights and thresholds, the prediction performance of the network system is poor. Therefore, the GA global optimization ability is used to find the best weights and thresholds, and the GA-ELM prediction model optimized by genetic algorithm is obtained. The trained GA-ELM network prediction system can better map the relationship between process parameters and quality indexes, and then use GA to optimize the GA-ELM network model to find the better process combination. Through orthogonal experimental design and range analysis, the author found out the influence degree of process parameters on the warpage deformation of the plastic part [7], and used the ELM model optimized by genetic algorithm to fit the nonlinear function relationship between warpage deformation and injection molding process parameters. Finally, genetic algorithm was used to optimize the GA-ELM network model after fitting to find out the small warping deformation, which solved the actual warping deformation of air conditioning bracket in injection molding to a certain extent.

\section{GA-ELM-GA optimization network model}

\subsection{ELM fundamentals}

ELM (Extreme Learning Machine) is a feedforward neural network model of a single hidden layer. The weight matrix $\mathrm{W}$ between the input layer and the hidden layer and the threshold matrix B of the hidden layer neurons are generated randomly. There is no need to modify the weight matrix $\mathrm{W}$ and threshold matrix $\mathrm{B}$ during the training process, and the unique optimal solution can be obtained by changing the number of neurons in the hidden layer.

\subsection{Build GA-ELM network prediction model}

Due to the random generation of the weight matrix w between the ELM input layer and the hidden layer and the threshold matrix $\mathrm{b}$ of the hidden layer neurons, the prediction fitting ability and prediction stability of the network model are insufficient. GA is an optimization algorithm that simulates the process of biological evolution and can avoid the shortcomings of traditional optimization algorithms such as local optimization and insufficient search 
ability. With efficient optimization ability. GA is used to optimize the weight matrix w and the threshold matrix $b$ of the neurons in the hidden layer to find the optimal $w$ and $b$. The optimal ELM network prediction system is obtained.

GA optimization ELM network model algorithm steps are as follows:

1) The training samples were extracted, the sample data were normalized, and necessary parameters of the ELM network model were set, such as the number of iterations, the size of the population, the number of neurons in the hidden layer, etc [8].

2) he global optimization ability of GA is used to find the best weight $w$ and threshold $\mathrm{b}$. In the population initialization stage, the weight $\mathrm{w}$ and threshold $\mathrm{b}$ are randomly selected, and the optimal $\mathrm{w}$ and $\mathrm{b}$ are obtained through continuous selection, crossover and mutation operations. The fitness function of GA is expressed by Equation (1):

$$
\text { error }=\frac{\sum_{i, j}\left|y_{i j}-x_{i j}\right|}{\mathrm{n}}
$$

In formula (1): error is the average error of the ELM model relative to the test set. $y_{i j}$ is the output of the ELM network model. $x_{i j}$ is the input for the ELM network model. $\mathrm{n}$ is the number of samples.

3) The weight and threshold values of GA optimization are transmitted back to the ELM network, and the model of the ELM network optimized by GA is obtained. The error between the predicted value and the true value is analyzed to check the prediction accuracy of the system. Through reference[9-10], the variable parameters were adjusted several times to meet the prediction model with better prediction effect, and a group of better recommended values were selected.

\subsection{Construction of GA-ELM-GA optimization model}

The steps of GA-ELM combined with genetic algorithm (GA) for extremum optimization are mainly divided into GA-ELM network model training fitting and GA-ELM network model training extremum optimization using genetic algorithm.

Proceed as follows[11-13]:

1) The steps of GA-ELM combined with genetic algorithm (GA) for extremum optimization are mainly divided into GA-ELM network model training fitting and GA-ELM network model training extremum optimization using genetic algorithm.

2) Genetic algorithm (GA) optimization part: Determine the optimization target Fmin, optimization variables $x_{i}$ and constraint range of the genetic algorithm. Use the powerful global optimization capability of genetic algorithm to find the minimum quality index and corresponding process parameters of the trained GA-ELM network model. The mathematical model of genetic algorithm established is as follows (2).

$$
\begin{aligned}
& \text { Fmin }=f\left(\mathrm{x}_{1}, \mathrm{x}_{2}, \ldots, \mathrm{x}_{\mathrm{i}}\right) \\
& \text { s.t. }\left\{\begin{array}{c}
a_{1} \leq \mathrm{x}_{1} \leq b_{1} \\
a_{2} \leq \mathrm{x}_{2} \leq b_{2} \\
\cdot \\
\cdot \\
\cdot \\
a_{i} \leq \mathrm{x}_{\mathrm{i}} \leq b_{i}
\end{array}\right.
\end{aligned}
$$


In formula (2): $a_{i}, b_{i}$ are the lower limit and upper limit of the constraint range of the optimization variable $\mathrm{x}_{i} \cdot f\left(\mathrm{x}_{1}, \mathrm{x}_{2}, \ldots, \mathrm{x}_{\mathrm{i}}\right)$ is the fitness function of the genetic algorithm. The predicted output of the trained GA-ELM network model is taken as the individual fitness value. According to the fitness value, the better individuals are screened and the worse ones are eliminated. Set the appropriate population size, crossover probability, mutation probability and the maximum number of iterations. Searching for the optimal individual through selection, crossover, mutation and other operations. The GA-ELM-GA model flow is shown in Figure 1.

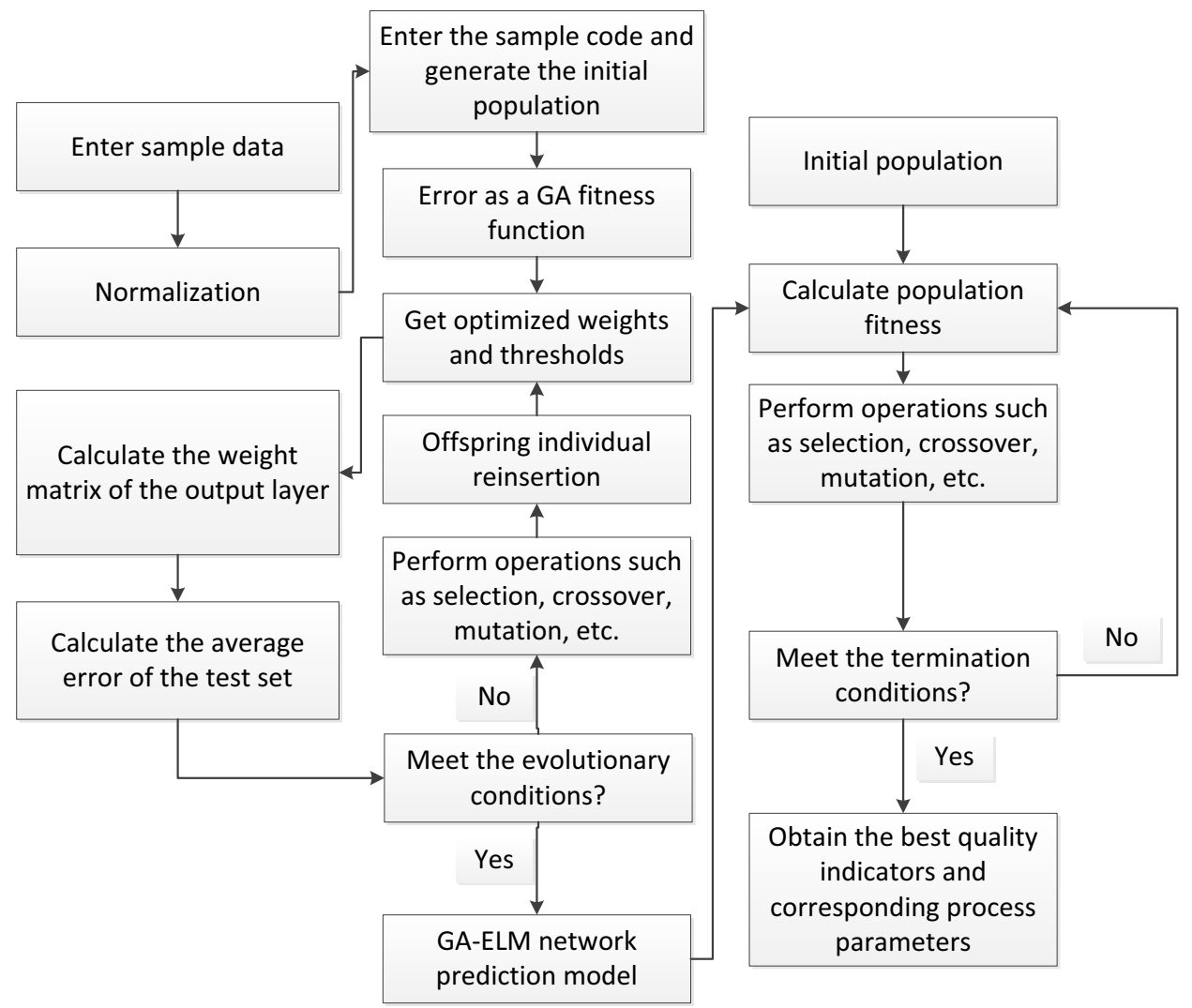

Fig. 1. GA-ELM-GA flow chart.

\section{Instance}

\subsection{Shell structure of electrical connector}

The basic size of the electrical connector shell is $137 \mathrm{~mm} \times 70 \mathrm{~mm} \times 18 \mathrm{~mm}$. As shown in Figure 2, there are many irregular areas inside, which are prone to warping and deformation in the injection molding process. The maximum wall thickness measured by NX12.0 is $3 \mathrm{~mm}$. The average wall thickness was $1.5 \mathrm{~mm}$. Electrical connector shell plastic parts used in electrical connection, in quality, appearance has higher requirements. This plastic part is thin-walled parts, easy to produce warp deformation, material selection of Monsantokasei TFX-210 acrylonitrile-butadiene-styrene copolymer (ABS), ABS is a kind of 
comprehensive performance of the material, the surface is smooth, has very high wear resistance and other advantages. The plastic parts model was imported into Moldflow software for simulation analysis, and the two-layer mesh was set to divide the mesh. After repairing the mesh, the mesh statistics showed that the number of triangular units was 31396 , with the maximum aspect ratio of 15.18 , the minimum of 1.16 , and the average of 2.15. There were no incorrect alignment and completely overlapping units. The matching percentage is $91.4 \%$, which meets the condition of the model flow analysis.

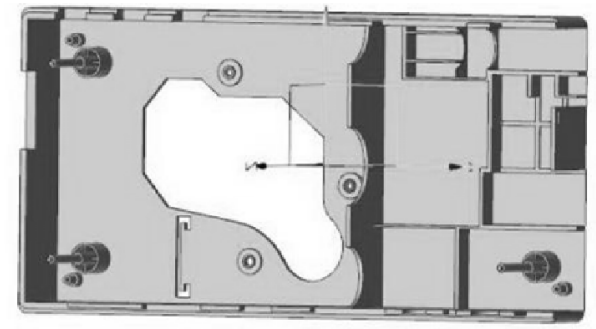

Fig. 2. Shell structure of electrical connector.

\subsection{Orthogonal experimental design}

The recommended mold temperature (A), melt temperature (B) and injection time (C) were obtained by combining the window analysis of plastic parts forming, and the appropriate level range of the three test factors was designed. Pressure holding time (E) and pressure holding (F) test factors were selected by experience [8]. The values of experimental factor levels in Table 1 were designed. The orthogonal experimental samples in Table 2 and the corresponding warpage deformation were obtained by simulation in Moldflow, and the warpage deformation in the fifth group was $0.3822 \mathrm{~mm}$.

Table 1. Value range of factor level.

\begin{tabular}{cccccc}
\hline $\begin{array}{l}\text { Factor } \\
\text { level }\end{array}$ & $\begin{array}{c}\text { Mold } \\
\text { temperature } \\
\mathrm{A}\left({ }^{\circ} \mathrm{C}\right)\end{array}$ & $\begin{array}{c}\text { Melt } \\
\text { temperature } \\
\mathrm{B}\left({ }^{\circ} \mathrm{C}\right)\end{array}$ & $\begin{array}{c}\text { Injection } \\
\text { time } \\
\mathrm{C}(\mathrm{s})\end{array}$ & $\begin{array}{c}\text { compress } \\
\text { time } \\
\mathrm{D}(\mathrm{s})\end{array}$ & $\begin{array}{c}\text { Holding } \\
\text { pressure } \\
\mathrm{E}(\mathrm{Mpa})\end{array}$ \\
\hline 1 & 60 & 270 & 0.2 & 4 & 60 \\
2 & 65 & 275 & 0.3 & 5 & 65 \\
3 & 70 & 280 & 0.4 & 6 & 70 \\
4 & 75 & 285 & 0.5 & 7 & 75 \\
5 & 80 & 290 & 0.6 & 8 & 80 \\
\hline
\end{tabular}

Table 2. Orthogonal test samples and warpage deformation.

\begin{tabular}{ccccccc}
\hline $\begin{array}{c}\text { Serial } \\
\text { number }\end{array}$ & $\begin{array}{c}\mathrm{A} \\
\left({ }^{\circ} \mathrm{C}\right)\end{array}$ & $\begin{array}{c}\mathrm{B} \\
\left({ }^{\circ} \mathrm{C}\right)\end{array}$ & $\begin{array}{c}\mathrm{C} \\
(\mathrm{s})\end{array}$ & $\begin{array}{c}\mathrm{D} \\
(\mathrm{s})\end{array}$ & $\begin{array}{c}\mathrm{E} \\
(\mathrm{Mpa})\end{array}$ & $\begin{array}{c}\text { Warpage } \\
(\mathrm{mm})\end{array}$ \\
\hline 1 & 60 & 270 & 0.2 & 4 & 60 & 0.8226 \\
2 & 60 & 275 & 0.3 & 5 & 65 & 0.6847 \\
3 & 60 & 280 & 0.4 & 6 & 70 & 0.5410 \\
4 & 60 & 285 & 0.5 & 7 & 75 & 0.4532 \\
5 & 60 & 290 & 0.6 & 8 & 80 & 0.3822 \\
6 & 65 & 270 & 0.3 & 6 & 75 & 0.5620 \\
7 & 65 & 275 & 0.4 & 7 & 80 & 0.4555 \\
8 & 65 & 280 & 0.5 & 8 & 60 & 0.4159 \\
9 & 65 & 285 & 0.6 & 4 & 65 & 0.7981 \\
10 & 65 & 290 & 0.2 & 5 & 70 & 0.7643 \\
\hline
\end{tabular}




\begin{tabular}{lllllll}
\hline 11 & 70 & 270 & 0.4 & 8 & 65 & 0.4150 \\
12 & 70 & 275 & 0.5 & 4 & 70 & 0.8192 \\
13 & 70 & 280 & 0.6 & 5 & 75 & 0.7027 \\
14 & 70 & 285 & 0.2 & 6 & 80 & 0.6683 \\
15 & 70 & 290 & 0.3 & 7 & 60 & 0.5278 \\
16 & 75 & 270 & 0.5 & 5 & 80 & 0.7282 \\
17 & 75 & 275 & 0.6 & 6 & 60 & 0.5849 \\
18 & 75 & 280 & 0.2 & 7 & 65 & 0.5457 \\
19 & 75 & 285 & 0.3 & 8 & 70 & 0.4626 \\
20 & 75 & 290 & 0.4 & 4 & 75 & 0.8870 \\
21 & 80 & 270 & 0.6 & 7 & 70 & 0.5123 \\
22 & 80 & 275 & 0.2 & 8 & 75 & 0.4653 \\
23 & 80 & 280 & 0.3 & 4 & 80 & 0.9109 \\
24 & 80 & 285 & 0.4 & 5 & 60 & 0.7692 \\
25 & 80 & 290 & 0.5 & 6 & 65 & 0.6681 \\
\hline
\end{tabular}

\subsection{Range analysis}

Range analysis can find out the influence of different factors on the experimental results. The larger the average range of each factor is, the more important this factor is and the higher the influence on the experimental results, and at the same time, a better process combination can be obtained [9]. Range analysis was carried out on the results obtained from Moldflow simulation orthogonal experiment design. According to the range analysis in Table 3, it can be intuitively seen that the influence of factor variables on the warping deformation is: holding time (D) $>$ mold temperature (A) $>$ injection time $(\mathrm{C})>$ melt temperature $(\mathrm{B})>$ holding pressure.

Table 3. Range analysis table.

\begin{tabular}{cccccc}
\hline Mean & $\mathrm{A}$ & $\mathrm{B}$ & $\mathrm{C}$ & $\mathrm{D}$ & $\mathrm{E}$ \\
$\mathrm{K} 1$ & 0.5767 & 0.6080 & 0.6532 & 0.8476 & 0.6241 \\
$\mathrm{~K} 2$ & 0.5992 & 0.6019 & 0.6296 & 0.7298 & 0.6223 \\
$\mathrm{~K} 3$ & 0.6266 & 0.6232 & 0.6135 & 0.6049 & 0.6199 \\
$\mathrm{~K} 4$ & 0.6417 & 0.6303 & 0.6169 & 0.4989 & 0.6140 \\
$\mathrm{~K} 5$ & 0.6652 & 0.6459 & 0.5960 & 0.4282 & 0.6290 \\
$\mathrm{R}$ & 0.0884 & 0.0440 & 0.0572 & 0.4194 & 0.0150 \\
Impact: big $\rightarrow$ small & \multicolumn{5}{c}{$\mathrm{D}>\mathrm{A}>\mathrm{C}>\mathrm{B}>\mathrm{E}$} \\
Optimal combination & $\mathrm{D}_{5} \mathrm{~A}_{1} \mathrm{C}_{5} \mathrm{~B}_{2} \mathrm{E}_{4}$ \\
\hline
\end{tabular}

In order to visually see the degree of influence of different factors on the amount of warpage and the optimal combination of factors, the value of the factor variable is taken as the abscissa, and the amount of warpage is taken as the ordinate, and the effect of each factor level on the warpage is obtained in Figure 3. Influence the trend. The value range of each factor variable corresponds to the greater the span of warpage deformation, the greater the degree of influence. It can be seen that the biggest influence on the warpage deformation of the plastic part is the holding time. With the increase of the holding time D, the warpage deformation decreases sharply; followed by the mold temperature $\mathrm{A}$, the warpage deformation of the plastic part and the mold The temperature $\mathrm{A}$ is in a negative correlation. As the mold temperature A increases, the warpage deformation decreases; as the injection time $\mathrm{C}$ increases, the warpage deformation first decreases, then stabilizes, and finally decreases; as the melt temperature B increases Large, the change of the warpage deformation first decreases and then increases; the packing pressure $\mathrm{D}$ has the least influence on the warpage deformation. With the increase of the packing pressure $\mathrm{D}$, the 
warpage deformation first changes steadily and then increases, The turning point is at the position of $75 \mathrm{MPa}$. The optimal combination parameters $\left(\mathrm{D}_{5} \mathrm{~A}_{1} \mathrm{C}_{5} \mathrm{~B}_{2} \mathrm{E}_{4}\right)$ can also be obtained as mold temperature $60{ }^{\circ} \mathrm{C}$, melt temperature $275^{\circ} \mathrm{C}$, injection time $0.6 \mathrm{~s}$, holding pressure time $8 \mathrm{~s}$, and holding pressure $75 \mathrm{MPa}$.

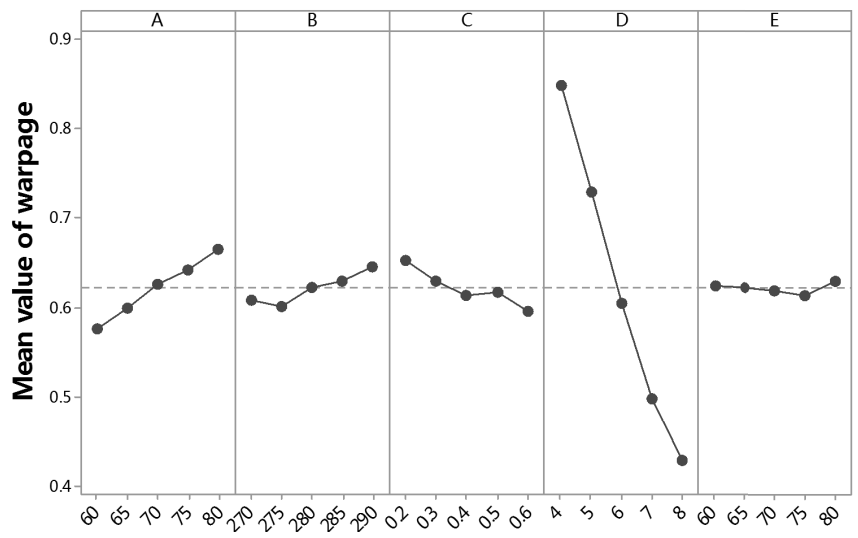

Fig. 3. Influence trend of each factor level on warpage deformation.

\section{Optimization of injection molding process parameters based on GA-ELM-GA algorithm}

The injection molding process parameters were optimized according to the GA-ELM network prediction model and GA-ELM-GA optimization model constructed above. 20 groups of random data in Table 2 were taken as training samples, and another 5 groups of data were taken as test samples to evaluate the prediction accuracy of GA-ELM network prediction model. The number of nodes in the input layer was set as 5, the number of nodes in the output layer was set as 1 , and the number of neurons in the hidden layer was set as 12 . In this way, the GA-ELM network prediction model trained by repeated training can be obtained, which can better map the nonlinear coupling relationship between the five process parameters and the warping deformation. Finally, GA is used to find the optimal process parameters in the GA-ELM network prediction model. The population size in the optimization model is set as 50 , and the cross probability is set as 0.5 . The probability of mutation is set to 0 . 4 . The maximum number of iterations is set to 100 .

\subsection{GA-ELM forecast results}

Figure 4 Comparison of the prediction results of the ELM and GA-ELM networks. It can be seen that when the ELM network model is directly used for prediction, $\mathrm{R}^{2}=0.79321$, the relative error is relatively large, and the prediction accuracy is insufficient. When the GAELM network model is used for prediction, $\mathrm{R}^{2}=0.99631, \mathrm{R}^{2}$ Close to 1 , the prediction accuracy is high, and the relative error is controlled within 2\%. This GA-ELM network model predicts the warpage deformation of the plastic part with high prediction accuracy and prediction efficiency. 


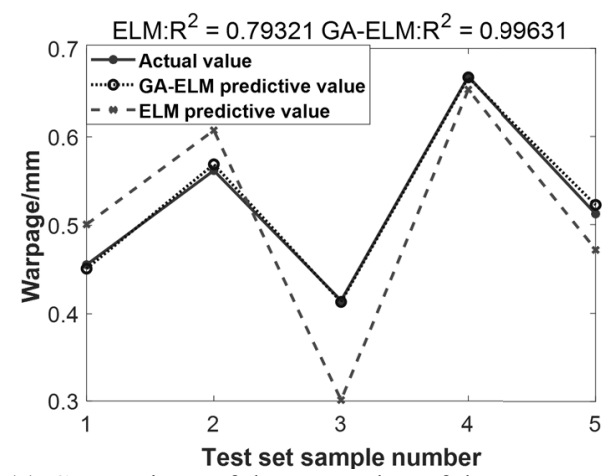

(a) Comparison of the true value of the test set with the predicted value of ELM and GA-ELM.

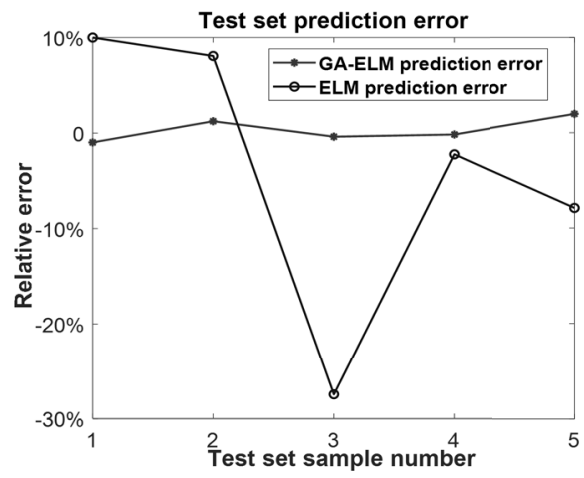

(b) ELM and GA-ELM network prediction relative error.

Fig. 4. Comparison of network prediction results between ELM and GA-ELM.

\subsection{GA-ELM-GA Search results}

GA is used to optimize GA-ELM by global extreme value. Figure 5 shows the fitness curve of GA optimization. After 100 iterations, the warping deformation of the plastic part converges to $0.2919 \mathrm{~mm}$, and the corresponding process parameters are mold temperature of $60.34^{\circ} \mathrm{C}$, melt temperature of $270.15^{\circ} \mathrm{C}$, injection time of $0.56 \mathrm{~s}$ and pressure holding time of $7.82 \mathrm{~s}$. The holding pressure is $75.23 \mathrm{MPa}$, and the predicted warpage deformation is $0.2919 \mathrm{~mm}$.

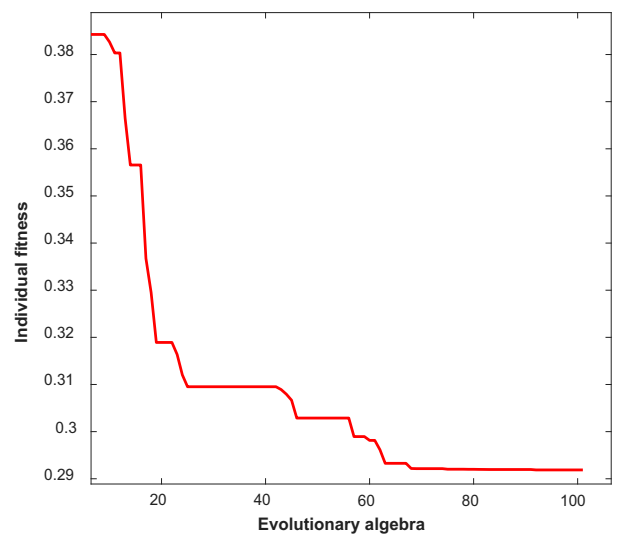

Fig. 5. Fitness curve.

\subsection{Simulation verification and optimization method comparison}

Five process parameters optimized by GA-ELM-GA were input into Moldflow for simulation verification, and the injection molding warpage cloud image was obtained. By comparing the warpage deformation predicted by GAELM-GA in Matlab with the simulated value of Moldflow $0.2981 \mathrm{~mm}$, the relative error between the two was $2.1 \%$. The reliability of GA-ELM network prediction system is verified again. Fig.6 shows the warpage deformation cloud images optimized by different optimization methods. It can be seen that the warpage deformation of $0.3566 \mathrm{~mm}$ optimized by range analysis decreases by $6.7 \%$ compared with the minimum warpage deformation of $0.3822 \mathrm{~mm}$ optimized by 
orthogonal test. The warp deformation of $0.2981 \mathrm{~mm}$ optimized by GA-ELM-GA is reduced by $22 \%$ compared with the minimum warp deformation of $0.3822 \mathrm{~mm}$ optimized by orthogonal test. The optimization effect is better than range analysis, and the injection molding quality of the plastic parts is improved to a great extent, which proves the reliability and superiority of this method.

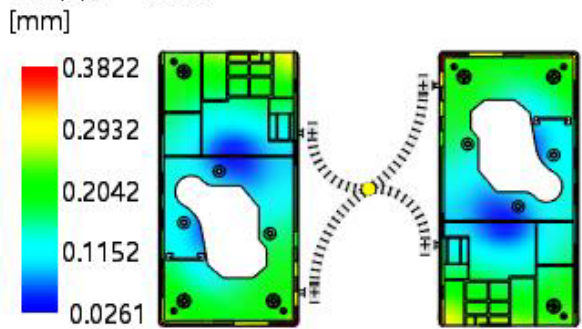

(a) Orthogonal test optimized minimum warpage deformation.

$[\mathrm{mm}]$

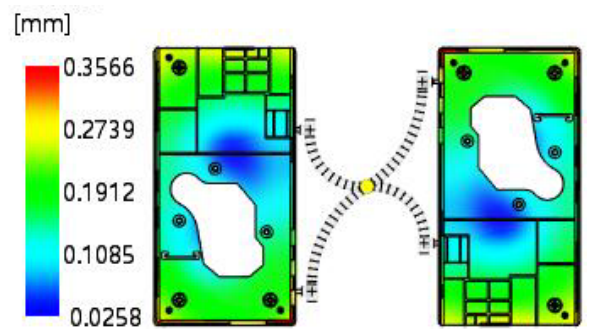

(b) Minimum warpage deformation optimized by range analysis.

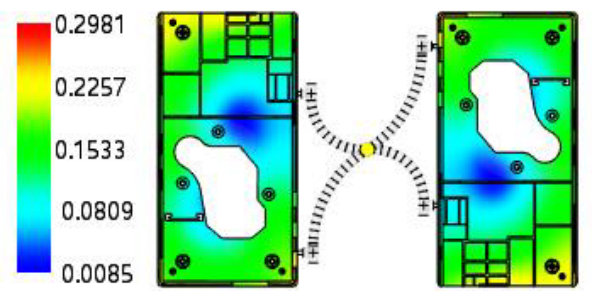

(c) GA-ELM-GA optimized minimum warpage deformation.

Fig. 6. The minimum warpage deformation optimized by the orthogonal experiment.

\section{Conclusion}

1) Taking the optimization of process parameters of injection molding parts as an example, orthogonal experimental design was designed. Moldflow was used to simulate and analyze the orthogonal test results, and the minimum warpage deformation optimized by orthogonal experiment was $0.3822 \mathrm{~mm}$. Through range analysis, the influence degree of factor variables (A, B, C, D, E) on the warping deformation was obtained as follows: holding time (D) $>$ mold temperature $(\mathrm{A})>$ injection time $(\mathrm{C})>$ melt temperature $(\mathrm{B})>$ holding pressure $(\mathrm{E})$. Meanwhile, the optimal combination $\mathrm{D}_{5} \mathrm{~A}_{1} \mathrm{C}_{5} \mathrm{~B}_{2} \mathrm{E}_{4}$ corresponding to range analysis was obtained, and the corresponding warping deformation was $0.3566 \mathrm{~mm}$.

2) Using GA to optimize the weights and thresholds of ELM, divide the samples obtained by the orthogonal experiment design and the corresponding warpage into a test set and a training set, and train the ELM model on the training set to obtain the GA-ELM network model. Through the test set, it can be obtained that the $\mathrm{R}^{2}$ of the ELM network model is 0.79321 , the $\mathrm{R}^{2}$ of the GA-ELM network model is 0.99631 , and the relative error of the GA-ELM network model prediction is small. This GAELM network model can be used for the plastic part warpage deformation prediction.

3) GA-ELM model was obtained through 2). GA was used to optimize the GA-ELM network model to obtain the optimized process parameters and corresponding warpage deformation. The relative error of $0.2981 \mathrm{~mm}$ warp deformation optimized by GAELM-GA and $0.2919 \mathrm{~mm}$ simulated by Moldflow is $2.1 \%$, which again verifies the reliability of GA-ELM prediction system, and it is reduced by $22 \%$ compared with the minimum warp deformation optimized by orthogonal test. To a great extent, the 
injection molding quality of electrical clasp shell is improved, and the optimization effect is better than that of range analysis and orthogonal experiment. It can provide a new optimization method for the following process personnel in the field of process parameter optimization.

\section{References}

1. Huang HL. Prediction of warpage deformation of two-color plastic parts based on BP neural network[J]. Plastics Technology,2019,47(06):68-71.

2. Wang, CF, Huang, M, Shen, CY,et al. Warpage prediction of the injection-molded strip-like plastic parts[J]. Chinese Journal of Chemical Engineering, 2016, 24(05):665670.

3. Sun LL, Su XM. Optimization design of injection mold for automobile built-in storage box based on MPI [J]. China Plastics, 2018,32(04):125-131.

4. Wang HX, Wang JL, Huang Qixin. Reverse engineering modeling and die design for the back cover of hair dryer handle [J]. China Plastics, 2018,32(11):131-135.

5. Jiang AY, Zhang BF, Jia ZH,et al. Injection molding process optimization of long glass fiber reinforced polypropylene composites [J]. Plastics, 2020,49 (03): 85-89

6. Sheng LJ. Research on Optimization of molding process of automotive sound plastic parts based on BP neural network[J]. Plastics Technology, 2018, 46(11): 44-48.

7. Wang YL. Baltic dry bulk freight index forecast model based on GA-ELM [J]. China Logistics and Purchasing, 2019(22): 37-38.

8. Mei Y, Song PY, Chen LY, et al. Research and application of ga-elm based vibration prediction model for manipulator [J]. Computer simulation, 2019,36 (09): 352-356

9. Ji N, Zhang WX, Yu YY, et al. Multi-objective optimization of injection molding based on optimal Latin hypercube sampling method and NSGA- II algorithm[J]. Engineering Plastics Application, 2020, 48(03): 72-77 .

10. Li JB, Yang QF. Study on injection molding process of POM oil pump bracket flange for automobile [J]. Application of Engineering Plastics, 2010(11): 39-42.

11. Liu Q, Chen HR, Mei D, et al. Optimization of injection molding process parameters of navigation lights based on orthogonal experiment and BPNN-GA[J]. Journal of Plasticity Engineering, 2020, 27(07): 123-129.

12. Ni HF, Si LT, Huang JP, et al. The extreme learning machine optimized by nearinfrared spectroscopy and genetic algorithm realizes the rapid determination of effective ingredients in the purification process of Ginkgo biloba[J]. Chinese Journal of Chinese Materia Medica, 2021, 46(01): 110- 117.

13. Mei Y, Liu HB, Luo NK, et al. Analysis and prediction of die wear during precision forging of titanium alloy blades based on GA-ELM[J]. Forging Technology,2020,45(10):130-136.

14. Zhang LB, Huang HS, Yao LG. Optimization of injection molding process parameters based on BP neural network and MIV algorithm[J]. Plastics Technology, 2018, 46(12): 94-99

15. Liu RJ, Zhang YW, Wen CW, et al. Orthogonal experimental design and analysis method research [J]. Experimental technology and management, 2010,27 (09): 52-55. 